\title{
Muscle Fatigue Monitor: A Noninvasive Device for Observing Localized Muscular Fatigue
}

\author{
FOSTER B. STULEN, MEMBER, IEEE, AND CARLO J. DE LUCA, SENIOR MEMBER, IEEE
}

\begin{abstract}
As a muscular contraction is sustained, the spectrum of the myoelectric signal is compressed into lower frequencies. This spectral compression has been associated with localized muscular fatigue by several investigators. A device is presented that implements a technique to track the spectral compression by calculating the median frequency and two other parameters of the spectrum. The device is referred to as the muscle fatigue monitor (MFM) and is built with analog circuitry so that parameters are calculated and displayed in real-time and on-line. The technique is based on modulated filters which are implemented by using periodically-controlled switches to effectively vary the cutoff frequencies of the filters. The median frequency of a myoelectric signal obtained during a sustained, constant-force, isometric contraction was calculated by the MFM and digital computation. The results obtained by the two techniques were essentially the same, verifying the operation of the MFM.

Several other techniques to quantify the spectral compression are discussed and compared to the MFM. While most of the other techniques do track spectral compression, the features of the MFM make it appropriate and accurate for clinical and industrial applications.
\end{abstract}

\section{INTRODUCTION}

W HEN a muscle contracts, an electrical signal known as the myoelectric (ME) signal can be detected inside and/or outside the muscle. Since 1912, the frequency content of the ME signal has been known to shift into lower frequencies as a voluntary muscular contraction progresses to exhaustion [1]. Many investigators have confirmed the spectral shift of the ME signal [2]-[9].

In 1970, Lindström et al. [6] developed a mathematical model which expressed the power density spectrum of an ME signal obtained from a differential pair of surface electrodes, oriented parallel to the muscle fibers, as a function of the conduction velocity of the muscle fibers. They mathematically demonstrated that the conduction velocity of the muscle fibers scales the power density spectrum of the ME signal, thereby indicating that the previously considered frequency shift towards the low-frequency end is actually a spectral compression. Although other factors may conceivably influence the fre-

Manuscript received July 14, 1981 ; revised April 29, 1982. This work was supported in part by the National Institute for Handicapped Research under Grant 23-P-55854, by the Department of Education, HarvardMIT Program in HeaIth Science and Technology, by the National Institutes of Health under a Research Grant to MIT, and by the Liberty Mutual Insurance Company.

F. B. Stulen was with the NeuroMuscular Research Laboratory, Department of Orthopaedic Surgery, Children's Hospital Medical Center, Harvard Medical School, Boston, MA 02115. He is now with the Battelle Columbus Laboratories, Columbus, $\mathrm{OH} 43201$.

C. J. De Luca is with the NeuroMuscular Research Laboratory, Department of Orthopaedic Surgery, Children's Hospital Medical Center, Harvard Medical School, Boston, MA 02115. quency compression, conduction velocity decrease remains the most likely cause and is the best documented. In 1966, Stälberg [10] showed by direct measurements that the conduction velocity of muscle fibers decreased as voluntary muscular contractions progressed to exhaustion. More recently, Stulen [9] has obtained an empirical indication that the conduction velocity and the spectral compression of the ME signal are in fact directly related during a sustained electrical excitation of the muscle.

The decrease in conduction velocity has been attributed to the accumulation of metabolic by-products such as lactic acid [11]. The accumulation of metabolic by-products during a sustained contraction could be due to an increase in their production [12] and/or a decrease in their removal due to diminished blood flow during forceful contractions [13]-[15]. The accumulation of lactic acid and the subsequent lowering of muscle $\mathrm{pH}$ has long been associated with localized muscular fatigue [16], [17] and muscle pain [18]. Therefore, a reliable measure of the spectral compression could be used as a reliable measure of localized metabolic muscle fatigue.

In fact, the use of spectral compression as a measure of fatigue is gaining acceptance in neuromuscular clinics and in the evaluation of industrial work stations. Kadefors et al. [19], Herberts et al. [20], and Hagberg [21] have studied the effect of elevated arm positions on localized muscular fatigue in the shoulder muscles experienced by many workers. In the clinical environment, manual muscle tests are currently the primary procedure for determining muscular strength and endurance. However, the accuracy of these tests depends upon the training, skill, and experience of the clinician performing the examination [22], [23]. A convenient, objective, quantitative approach to determine changes in the state of the muscle is now possible with the spectral compression. Bellemare and Grassino [24], Gross et al. [25], [26], Schweitzer et al. [27], and Solomon et al. [28] have studied fatigue in the human diaphragm. Solomon et al. [28] used the spectral compression to set the resistance to breathing to safely exercise the diaphragm in quadriplegic patients.

Most current techniques to quantify the spectral compression use digital computations of the spectrum. The spectrum is time dependent due to the compression; hence, it must be determined over various intervals during the contraction. Once obtained, characteristic frequencies such as the mode, median, mean, or other parameters can be determined and used to measure the spectral compression [29].

In this paper, the development of a noninvasive device, the muscle fatigue monitor (MFM) is presented. This device mea- 


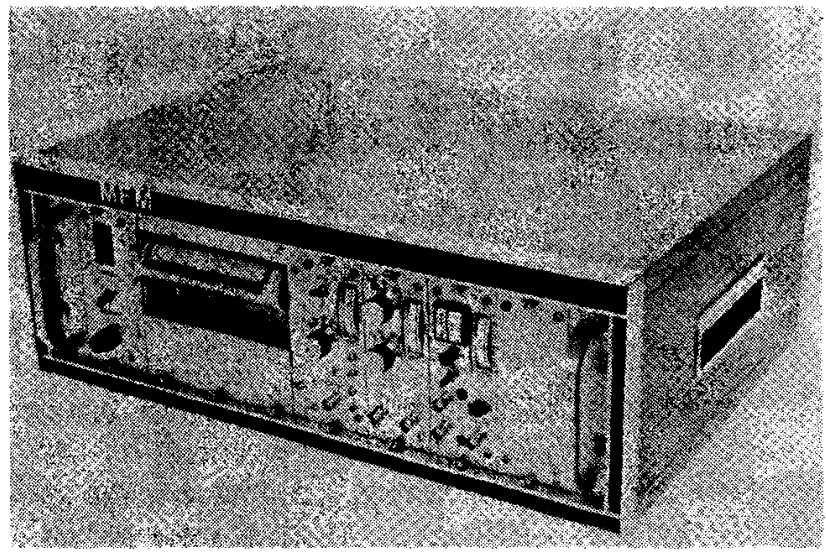

Fig. 1. The muscle fatigue monitor.

sures the spectral compression and therefore, the localized muscular fatigue, by calculating the median frequency and two other parameters of the spectrum. This signal processing is performed on-line and in real time with analog hardware [9] , [30], [31]. The median frequency was chosen as the parameter for measuring the frequency compression because according to Stulen and De Luca [29], it is often a more reliable estimator than other convenient parameters such as the mean or mode frequencies. The laboratory prototype of the MFM is shown in Fig. 1.

\section{Principles of Operation}

The technique used in the MFM is schematically represented in the block diagram of Fig. 2.

The ME signal that is obtained from differential electrodes is amplified and bandpass filtered ( $3 \mathrm{~dB}$ points at $20 \mathrm{~Hz}$ to 1.0 $\mathrm{kHz}$ ) to remove $\mathrm{dc}$ offsets, low-frequency artifacts associated with body movements, and high-frequency noise components. The amplified signal is simultaneously passed through highpass and low-pass filters whose cutoff frequencies are designed to be equal and to be modulated by a control voltage. The filters have sharp roll-offs $(60 \mathrm{~dB} /$ octave) in their respective rejection bands. Hence, for practical purposes, the two resulting signals may be considered orthogonal.

Each signal is then passed through a circuit that calculates the true rms voltage. (The circuits which calculate the rms voltages could be replaced with any circuit which converts an ac signal to a dc voltage corresponding to a measure of the spectral density; e.g., the logarithm of the rms voltage, the mean-squared voltage, or its logarithm.) The low-rms and highrms voltages are connected to control circuitry. The initial stage calculates and amplifies the difference in the rms voltages. The last stage of the circuit employs an integrator whose output is connected to a sample-and-hold circuit, which is initially kept in the sample state. The output of the sample-and-hold is then connected to the circuit which modulates the cutoff frequency of the filters. The difference of the low-rms and high-rms voltages varies the output of the integrator which adjusts the cutoff frequency of the filters to drive the difference in the rms voltages to zero. In other words, the power contained in the signals above and below the cutoff frequency

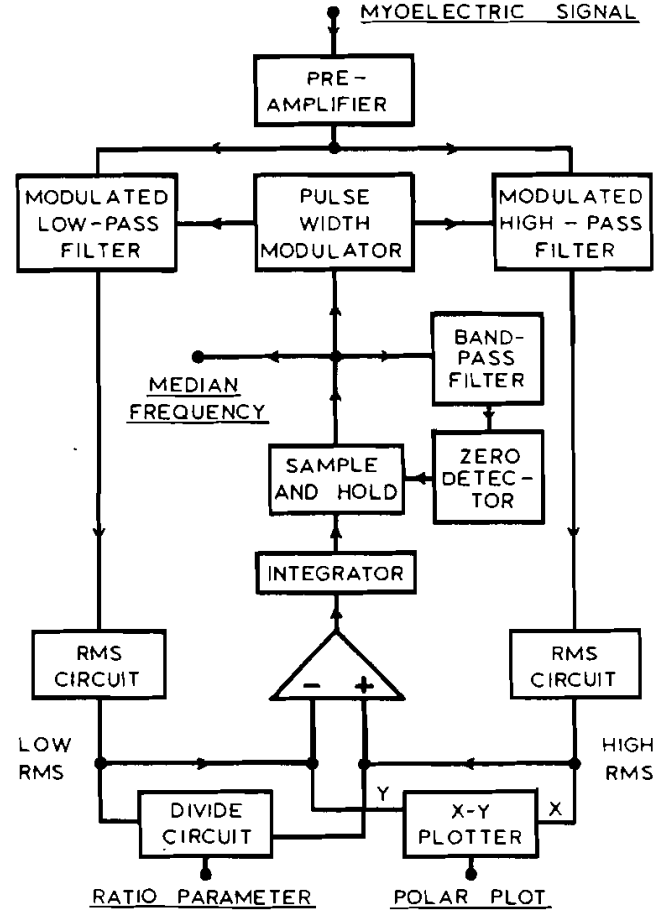

Fig. 2. Block diagram of the technique used in the muscle fatigue monitor (U.S. Patent 4213 467).

is equal. This is the definition of the median frequency. Thus, at this point, the integrator output uniquely corresponds to the median frequency of the power spectral density of the ME signal.

In one mode of operation, referred to as the track mode, the sample-and-hold circuit is maintained in the sample state. Hence, the integrator output continuously adjusts the cutoff frequency to the median frequency. Thus, the integrator output approximates a function of the time-dependent median frequency. Fig. 3 shows an ME signal obtained during a sustained isometric, constant-force contraction of the deltoid performed at 50 percent of maximal voluntary contraction. In the track mode of the MFM, the median frequency of the ME signal decreases initially and eventually levels off, as observed in Fig. 3(a).

In the second mode of operation, referred to as the hold mode, two additional parameters of the spectrum are obtained. The sample-and-hold circuit is initially fixed in the sample state. During the initial "seeking" for the median frequency, there are relatively large, rapid variations in the integrator output. These variations are detected and used to determine when the estimate approaches the actual value of the median frequency. At this point, the sample-and-hold circuit is switched to the hold mode. Thus, the cutoff frequency is held at the initial estimate of the median frequency. Two parameters may then be obtained in this mode. The first parameter, known as the polar parameter, is a plot of the low-rms voltage versus the high-rms voltage [32]. The parameter yields a polar representation of the rms voltage of the ME signal [shown in Fig. 3(b)] . The deviation of the curve from the $45^{\circ}$ line in a counterclockwise direction is consistent with the spectral compression of the ME signal. This parameter has a drawback because it is 

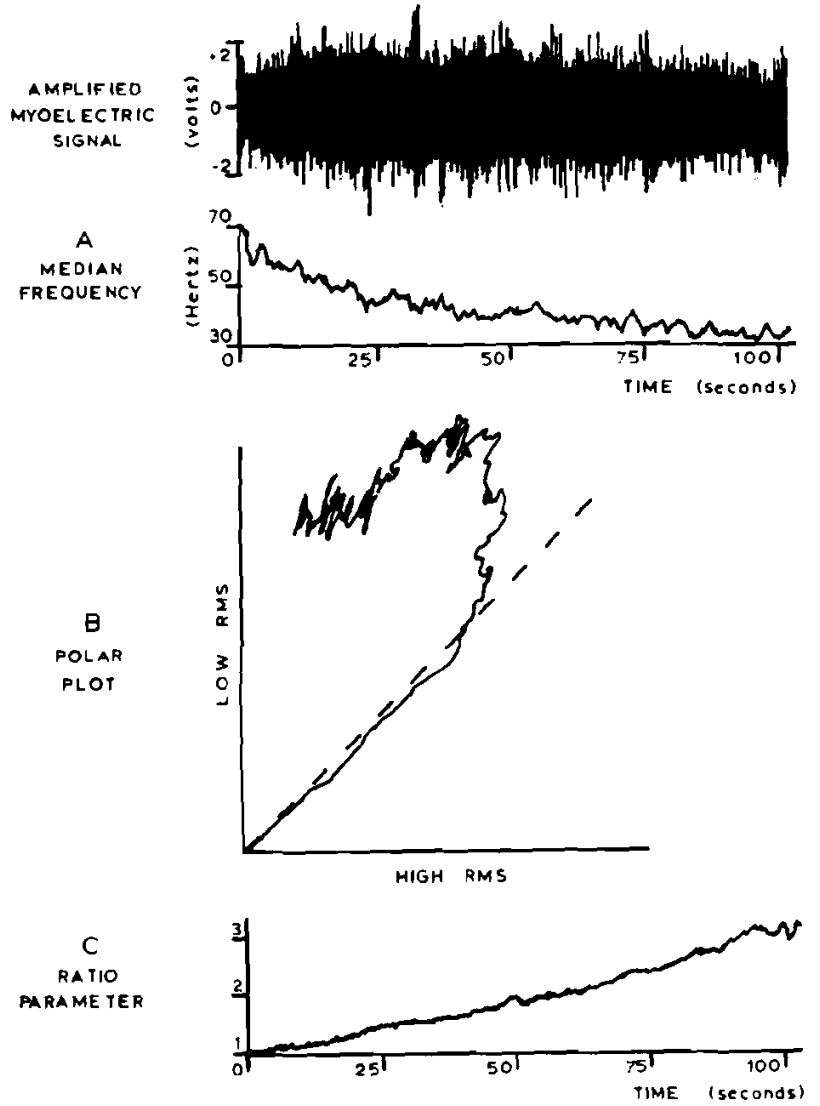

Fig. 3. A myoelectric signal obtained during a sustained, isometric, constant-force contraction at 50 percent of maximal level, and the outputs of the muscle fatigue monitor.

not necessarily a monotonic function of time. The second parameter of the hold mode, the ratio parameter [presented in Fig. 3(c)], overcomes this limitation. This is a plot of the ratio of the low rms to high rms voltage as a function of contraction time.

A variation of the technique of the MFM is depicted in Fig. 4. In this approach, the whole-rms voltage is multiplied by $\sqrt{1 / 2}$ and is then compared to the low-rms voltage [31], [33]. The integrator output only adjusts the cutoff frequency of the low-pass filter, greatly simplifying the circuit. Another advantage is that it not only yields the spectral compression information by the median frequency, but also produces the $\mathrm{rms}$ amplitude of the whole ME signal. However, the control law is not symmetric, causing the tracking to be faster when the estimate of the median frequency is below the actual value, as compared to when it is above. Nonetheless, the reduction in circuitry and the simultaneous calculation of frequency and amplitude parameters make this method well suited for clinical use.

\section{ImPlementation of the Modulated Filters}

The major feature of the MFM is the modulation of the cutoff frequency of the two filters. This was accomplished by using the "switched resistor" technique to vary the resistancecapacitance $(R C)$ product of the filters. This was chosen over others because it is particularly well suited to operate in the low-frequency range of the ME signal and is readily adaptable to the multipole filters required in the implementation. Franks and Sandberg [34] used a similar method to analyze a commutated capacitor as an $N$-path filter and to simulate transmission lines. Other applications have been in commutated networks, chopper networks of transmissions lines, and amplification of circuit transfer functions [35]-[39] .

The technique may be described by considering the singlepole, low-pass, $R C$ filter shown in Fig. 5(a). In this example, the resistor is switched in and out of the circuit at a constant frequency. The period in which the resistor is conducting in the cycle is known as the on-time. The on-time divided by the period of the switching operation is known as the duty cycle $n$. The signal controlling the switch $[y(t)]$ is a pulse width modulated signal. When $y(t)$ is high, the switch is on and current flows through the resistor. When $y(t)$ is low, the switch is open and no current flows. If $y(t)$ is modeled to have a value of 1 when high and 0 when low, then the Kirchhoff Current Law at node $a$ may be expressed as

$$
\frac{v_{i}(t)-v_{o}(t)}{R} \cdot y(t)=C \frac{d\left[v_{o}(t)\right]}{d t}
$$

where $v_{i}(t)$ is the input signal and $v_{o}(t)$ is the output signal at node $a$. The variable $t$ denotes time. The Fourier transform of (1) is expressed as

$$
\frac{\left[V_{i}(f)-V_{o}(f)\right]}{R} * Y(f)=j 2 \pi f C V_{o}(f) \text {. }
$$

The variables expressed with capital letters are the Fourier transforms of the time domain variables in lower case. The $f$ denotes frequency. The Fourier transform of $y(t)$ may be expressed as a weighted sum of Dirac delta impulses occurring at multiples of the switching frequency $F$. Therefore, it can be shown that the Fourier transform of $v_{o}(t)$ is

$$
\begin{aligned}
V_{o}= & \frac{1}{1+(2 \pi R C / n) j f} V_{i}(f)+\frac{1}{1+(2 \pi R C / n) j f} \\
& \cdot \sum_{\substack{k=-\infty \\
\neq o}}^{\infty} \operatorname{sinc}(n k)\left[V_{i}(f-k F)-V_{o}(f-k F)\right] .
\end{aligned}
$$

This expression may be simplified to a linear transfer function if the cutoff frequency of the $R C$ combination, $2 \pi R C$, is much less than the switching frequency $F$ :

$$
\frac{V_{o}(f)}{V_{i}(f)}=\frac{1}{1+(2 \pi R C / n) \cdot j f} .
$$

From the above transfer function, it may be seen that the cutoff frequency $f_{c}$ is proportional to the duty cycle of the switching function $y(t)$ :

$$
f_{c}=\frac{n}{2 \pi R C}
$$

Since the duty cycle is limited to values between 0 and 1 , the cutoff frequency may be adjusted between 0 and the frequency determined by the $R C$ product:

$$
0 \leqslant f_{c} \leqslant \frac{1}{2 \pi R C}
$$




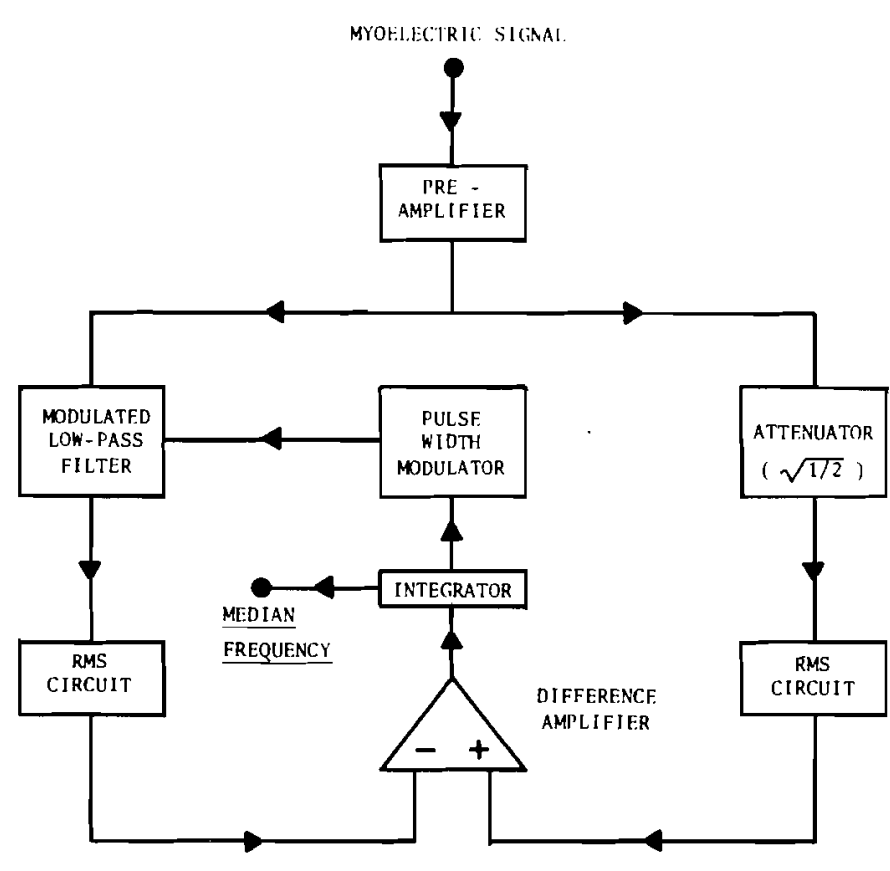

Fig. 4. Block diagram of a variation of the technique used in the muscle fatigue monitor (U.S. Patent 4213 466).

This formulation may also be used to calculate the Kirchhoff Current Law for the single-pole, high-pass filter shown in Fig. 5(b). An analogous expression to (3) may be derived for the Fourier transform of $v_{o}(t)$ :

$$
\begin{aligned}
V_{o}(f)= & \frac{(2 \pi R C / n) \cdot i f}{1+(2 \pi R C / n) \cdot i f} V_{i}(f)+\frac{(2 \pi R C / n) \cdot i f}{1+(2 \pi R C / n) \cdot i f} \\
& \cdot \sum_{\substack{k=-\infty \\
\neq 0}}^{\infty} \operatorname{sinc}(n k)\left[V_{i}(f-k F)-V_{o}(f-k F)\right] .
\end{aligned}
$$

In this case, a linear transfer function cannot be approximated under the previously made assumptions, because the sum of sinc terms is not passed through a low-pass filter as in (3). All artifacts associated with switching are passed unattenuated; therefore, a low-pass filter must follow the switched, high-pass filter in order to remove these artifacts. The cutoff frequency of the low-pass filter must be equal to or greater than the highest frequency of the signal and must be less than the switch. ing frequency. Under these conditions, it is possible to obtain an approximate linear transfer function:

$$
\frac{V_{o}(f)}{V_{i}(f)}=\frac{(2 \pi R C / n) \cdot i f}{1+(2 \pi R C / n) \cdot j f} .
$$

This is the transfer function of a single-pole, high-pass filter with cutoff frequency modulated by the duty cycle.

The above discussion applies only to single-pole filters. For a multipole filter, each resistor must be switched by the same duty cycle in order to have the cutoff frequency of the filter proportional to the duty cycle.

Ten-pole Butterworth filters were chosen. The high-pass filter was followed by a ten-pole low-pass Butterworth filter to remove switching artifacts. In general, characteristics of a filter are dependent on the values of the resistors and capacitors which determine the cutoff frequency. Thus, a switched

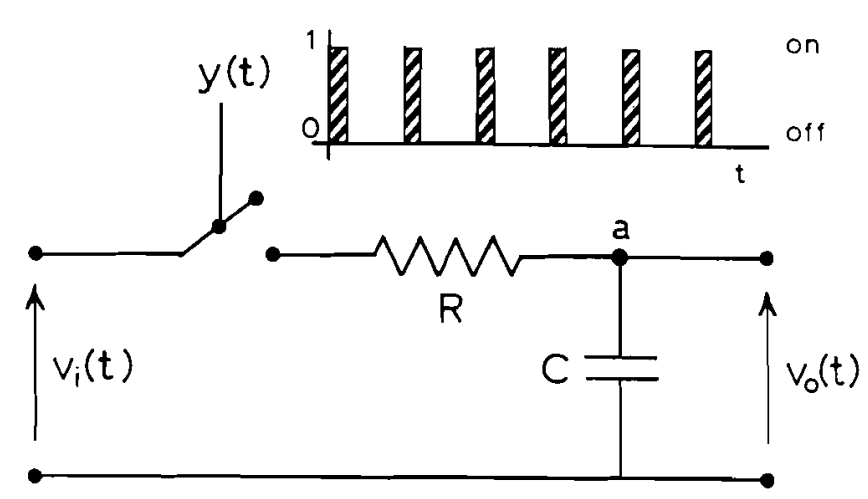

(a)

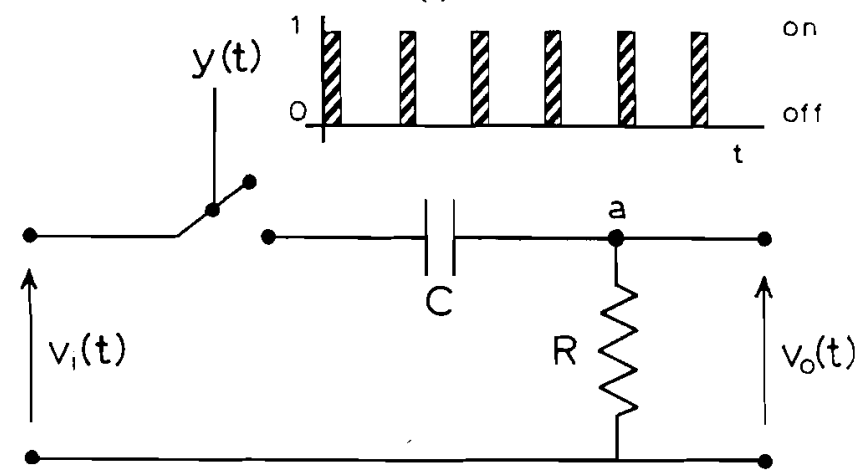

(b)

Fig. 5. (a) A single-pole, low-pass filter containing a periodically-operated switch. (b) A single-pole, high-pass filter containing a periodicallyoperated switch.

$R C$ filter may not have the same characteristics at various values of the duty cycle. For this reason, the modulated filter was constructed with operational amplifiers used as voltagecontrolled voltage sources. In this mode of operation, the values of the resistors and capacitors may be selected so that the filter characteristics are independent of the cutoff frequency. Hence, a switched Butterworth filter retains its characteristics independent of duty cycle.

The resistor and capacitor values were chosen to be $5.6 \mathrm{k} \Omega$ and $0.1 \mu \mathrm{F}$. The analog switches used in the circuit have a small but significant on-resistance of about $100 \Omega$. Thus, according to (6), the median frequency may be tracked from 0 to 257 $\mathrm{Hz}$. Hogan [40] and others have shown that for practical purposes, the entire bandwidth of an ME signal recorded with surface electrodes is limited to $300 \mathrm{~Hz}$. Therefore, the range of the median frequency is more than adequate.

\section{Modeling the Muscle Fatigue Monitor and Optimization of Control Parameters}

If the input signal is stationary, then the performance of the MFM must merely be stable for the output of the MFM to correspond to the median frequency of the input signal in steady state. However, when the input signal is nonstationary, the dynamic performance of the MFM limits its ability to track changes in the median frequency. If the variations in the median frequency are faster than the dynamic response of the MFM, then the output will not correspond to the median frequency. The performance of the MFM depends upon several factors: the stochastic properties of the input signal, the types of filters, the time constants of the rms circuits, and the gains of the control circuitry. 
To analyze the performance of the MFM, it was necessary to model the system. The circuitry was extensive and therefore, a component-by-component model would contain many state variables (on the order of $\mathbf{4 0}$ states). Two main assumptions were made to simplify the model: the filters were ideal with equal cutoff frequencies; and the filters responded instantaneously to a change in the control signal. The first assumption was justified because the filters were ten-pole Butterworth filters which were extremely flat in their passband and had very sharp roll-offs. The second assumption was a consideration of the switching frequency, which was at least an order of magnitude greater than the highest frequency component of the signal. The switching frequency determined the rate of modulation; thus, the dynamics of modulation were negligible compared to the slower dynamics of the signal passing through the filter. The details of the analysis are included in the Appendix.

An expression (A5) for the Laplace transform of the timedependent estimate of the median frequency $\hat{f}_{\text {med }}$ is derived in the Appendix and is presented here: From this equation, one notes that the MFM behaves as a second-order system. The steady-state error for the model of the MFM is zero, which can be determined by applying the final value theorem. The speed of response of the MFM was optimized by minimizing the following performance index:

$\hat{F}_{\text {med }}(s)=\frac{\left(A^{2} K_{c} / 2 \tau_{I} \tau_{R}\right)\left(K_{p}-K_{D} s\right) F_{k}(s)+\left(s+1 / \tau_{R}\right) \hat{f}_{\text {med }}^{o}}{s^{2}+\left[\left(1 / \tau_{R}\right)-\left(A^{2} K_{c} K_{D} / \tau_{I} \tau_{R}\right)\right] s+A^{2} K_{c} K_{p} / \tau_{I} \tau_{R}}$

where $f_{\text {med }}$ is the median frequency of the input signal which was assumed to have a step change at time equals zero. The purpose of the optimization was to select the gains of the difference amplifier $K_{p}$ and the differentiator $K_{D}$ as functions of the time constant of the rms circuit, $\tau_{R}$, to minimize the PI. An optimization computer program was used and the following values were determined: $K_{p}=44.7 / \tau_{R}$ and $K_{D}=0.472 \tau_{R}$. These values are optimal for the particular signal chosen. The optimal values will depend upon the actual signal characteristics and amplitude; however, the model signal is representative of signal amplitudes and bandwidths of actual ME signals (see the Appendix). Thus, the gains were set at these values.

Four time constants $(0.1,0.5,2.5$, and $10.0 \mathrm{~s})$ are switchselectable from the front panel. The switch also selects the values for the optimal control circuitry gains since the above analysis showed that they are functions of the time constant. The shorter time constants are used for forceful, exhausting contractions or when the median frequency is changing rapidly. The longer time constants are used for less exhaustive contractions or when average trends in periodic contractions are to be observed.

\section{Performance of the Muscle Fatigue Monitor}

A sine wave with variable frequency between 25 and $250 \mathrm{~Hz}$ was fed to the input of the MFM. The duty cycle of the pulse width modulator was measured directly, and the output of the MFM was observed. A plot of the frequency determined by the MFM as a function of the duty cycle is shown in Fig. 6 . The curves are shown for both versions of the MFM with time

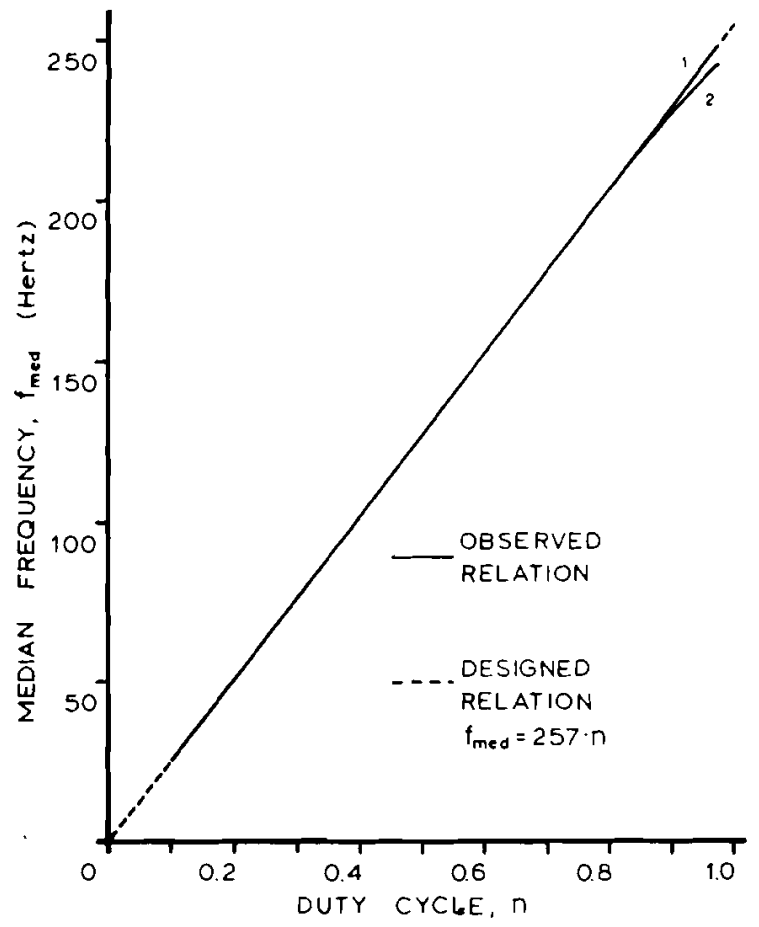

Fig. 6. Median frequency obtained with the muscle fatigue monitor versus the duty cycle of the switches of the modulated filters when the input is a sine wave. The line denoted by 1 relates to the version in Fig. 2 ; that denoted by 2 relates to the version in Fig. 4.

constants of $0.1,0.5$, and $2.5 \mathrm{~s}$. Both versions behaved in a linear fashion for values of the duty cycle between 0.1 to 0.8 as predicted by the analysis of the switched resistors. The version with the modulated high-pass and low-pass filters [30] represented in Fig. 2 continued to operate linearly between values of 0.8 and 1.0 . The version with only the modulated low-pass filter [31], [33] represented in Fig. 4 deviated from a straight line, indicating a lower frequency than the frequency of the input signal in this region.

The median frequency was determined by both the MFM and digital computation for an ME signal obtained during a sustained isometric constant-force contraction. The ME signal was connected to the MFM and both the output of the MFM and the ME signal were sampled by a PDP 11/34 computer. The power density spectrum of the sampled ME signal was calculated digitally using a fast Fourier transform algorithm. The median frequency was then calculated directly from the spectrum. As seen in Fig. 7, the estimates of the median frequency determined by the MFM and digital computation were essentially equal.

Fig. 8 contains three curves of median frequency versus contraction time. The curves were calculated with the MFM. The ME signals were obtained from the first dorsal interosseous muscle of a normal adult male with a pasteless differential surface electrode similar to that described by De Luca $e t$ al. [41] with an interelectrode distance of $10 \mathrm{~mm}$. The contractions were performed at 20,50 , and 80 percent of maximal voluntary contraction (MVC). The curves of the median frequency decreased as time progressed, demonstrating the ability of the MFM to track the spectral compression of the ME signal on-line and in real time. Even for the rapid change in the signal at 80 percent MVC, the MFM was able to quickly locate and track the median frequency. 


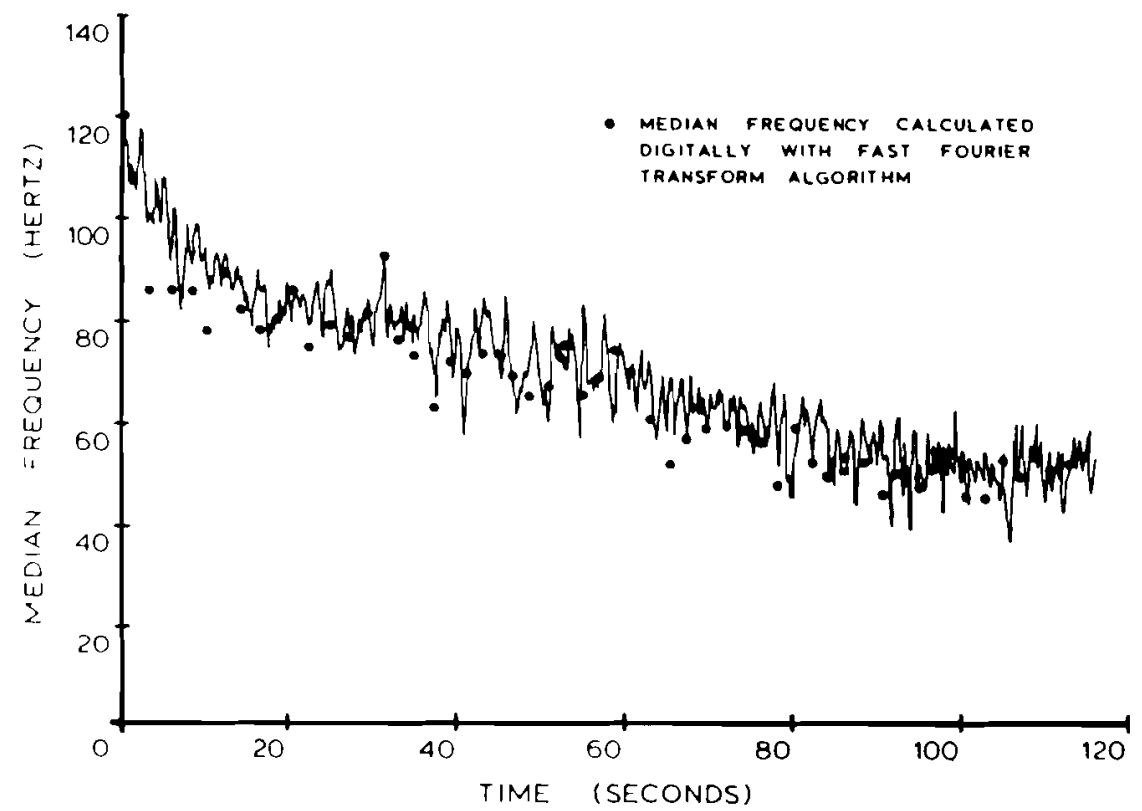

Fig. 7. Comparison of the median frequencies determined by digital computations and the muscle fatigue monitor.
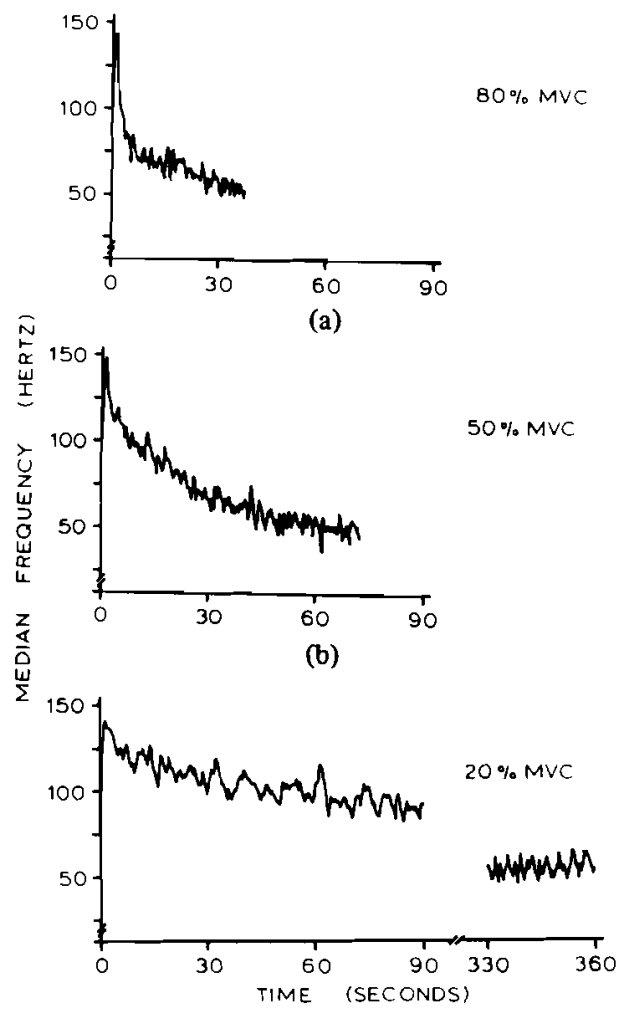

(c)

Fig. 8. Median frequency curves determined with the muscle fatigue monitor for sustained, isometric, constant-force contractions at (a) 80 , (b) 50 , and (c) 20 percent of the maximal level (MVC).

\section{Discussion}

Several investigators have tried various techniques to measure and quantify the spectral compression and the decrease in conduction velocity.

Stålberg [10] used a needle electrode unit specially constructed to observe action potentials from individual muscle fibers. Although this invasive technique directly measures the conduction velocity of individual muscle fibers, it does not provide an overall measure for all the active fibers. Further- more, the required needle alignment procedures are tedious, requiring accurate and secure electrode positioning. Needle electrodes are susceptible to small movements which could lead to large errors [42]. Thus, while this technique yields direct results, it involves complicated procedures, and would generally be limited to research investigations.

Kadefors et al. [4] passed the ME signal through four singleoctave bandpass filters. The frequency bands were chosen arbitrarily within the bandwidth of the signal. Each output of the filters was connected to a circuit which calculated the rms voltage. Their results were expressed in terms of the four timedependent voltages. Although this technique is sensitive to the spectral compression of the ME signal, it is somewhat cumbersome. The technique generates four parameters to quantify a single physiological phenomenon.

Lindström et al. [6], by mathematically modeling the power spectral density of the ME signal, were able to predict the occurrence of "dips" in the power density spectrum of an ME signal detected with differential electrodes. They proceeded to verify the presence of these dips at multiples of the inverse of the conduction velocity and in fact, calculated the average conduction velocity of the muscle fibers. Their results also showed that conduction velocity decreases during a sustained contraction. Although this technique is effective, it is very time consuming, requiring the calculation of the power density spectrum which is sufficiently smooth to determine the location of the dips. Since the conduction velocity is continuously changing, this procedure must be repeated for several time intervals of the ME signal.

In a more recent study, Lindström et al. [7] used a different technique to obtain an approximate measure of conduction velocity. It required the digital computation of the power spectral density in order to calculate the zero and first moments of the spectrum. The ratio of the first to the zero moment is the mean frequency. They showed that the conduction velocity is equal to the mean frequency times the spacing between the electrodes. Although this technique does yield an estimate of the conduction velocity directly, the 
digital computation is time consuming and cannot always be performed in real time.

Broman and Kadefors [43] developed an analog device that calculates the mean frequency of the power density spectrum for measuring the spectral compression. While the technique provides significant improvement over previous versions by yielding on-line, real-time processing, it requires a series of rational filters to approximte a $3 \mathrm{~dB} /$ octave filter. The accuracy of the estimate changes as the spectrum shifts due to this approximation, although for most applications, the provided accuracy may be sufficient.

Petrofsky [44] devised a series of 10 bandpass filters in the range of 35 to $350 \mathrm{~Hz}$ to measure some frequency components of the ME signal. The median frequency was then calculated with an analog computer. The estimate of the median frequency obtained in this fashion is limited in accuracy by the discrete number of filters used, although in practice, the accuracy of the estimate obtained may be acceptable for some applications.

Lynn [45] used an entirely different approach and developed a technique employing two pairs of differential surface ME electrodes which directly calculates the average conduction velocity of the muscle fibers. The electrodes are mounted in-line on the same base with an overall length of $30 \mathrm{~mm}$. This size precludes its use on several superficial muscles, such as those in the hands. The two ME signals are bandpass filtered with a narrow filter centered around $80 \mathrm{~Hz}$. This frequency corresponds to approximately the mode of an ME signal obtained at rest. Because of the spectral compression during a sustained contraction, the mode frequency may decrease to a third of this value. Therefore, the signal-to-noise ratio in the passband of the filter will decrease. Hence, the accuracy of the estimate of conduction velocity will degrade during a sustained contraction.

The MFM overcomes the drawbacks of some of the previous techniques. It uses only one pair of differential surface electrodes to obtain the ME signal. The processing is performed with analog hardware to yield on-line and real-time processing. The circuitry is optimized to maximize the speed of response so that cven very rapid changes in the frequency spectrum can be tracked. It specifically tracks the median frequency of the power density spectrum to measure the spectral compression of the ME signal during sustained muscle contraction. Stulen and De Luca [29] have shown that this is a preferred parameter for estimating the spectral compression. This allows the MFM to conveniently incorporate other types of devices that use the ME signal amplitude, such as some biofeedback devices. The circuitry is uncomplicated and reliable. These features imply that a handheld or portable unit can be constructed to be used routinely in clinical and industrial applications [31], [33]. This is a very important, but often overlooked consideration for the acceptance of a new technique and device.

\section{SUMMARY}

The muscle fatigue monitor is a noninvasive device which accurately estimates the median frequency and two other spectral parameters of the myoelectric signal. Even during a forceful contraction when the spectrum undergoes a rapid compression, the device can track and display the median frequency on-line and in real time. In addition to the spectral parameters, one implementation also determines the rootmean-squared value of the signal. All these features of the MFM provide convenience for monitoring the spectral compression as an indicator of localized muscular fatigue in clinical and industrial applications.

\section{APPENDIX}

The dynamics of the MFM are dependent upon the shape of the power density spectrum of the ME signal. A model signal was chosen for the analysis. The input signal was band-limited white noise because the average power contained in a region of the spectrum is merely a constant times the difference between the upper and lower frequencies. This greatly simplifies the calculations. Initially, the input signal had an upper frequency of $f_{h}$. The signal was applied for a sufficient time for the output of the MFM to equal the median frequency $f_{h} / 2$. At $t=0$, the upper frequency was halved to $f_{h} / 2$. The median frequency was thereby also halved to $f_{h} / 4$.

Each rms circuit was modeled as a single-pole, low-pass filter whose time constant equaled the time constant of the rms circuit. The input was considered to be the ensemble mean squared value and the output was the temporal mean squared value. The square root was replaced by a linear function. The point of the linearization was set at 1 . That is,

$$
f(x)=\sqrt{x}=\frac{1}{2}+\frac{1}{2} x+0(x)
$$

for $x$ in the neighborhood of 1 .

The amplitude of the signal was set so that the rms values were equal to 1 . At $t=0$, the signal amplitude was multiplied by 2 to keep the total signal power constant. Thus, when the output of the MFM equaled the new median frequency, both rms values equaled 1 again.

Although the change in amplitude was introduced to maintain the validity of a linear model, it had an empirical basis. The amplitude of ME signals obtained with surface electrodes during sustained, isometric, constant-force contractions is known to increase with contraction time [42]. Also, the model of Lindström et al. [6] shows that when the conduction velocity decreases, there is an increase in the surface $\mathrm{ME}$ signal amplitude in addition to the spectral compression.

Fig. 9 is a block diagram of the simplified dynamic model of the MFM. The power spectrum of the signal before and after $t=0$ is shown on the left of the figure.

The mathematical representation of the model is expressed as:

$$
\begin{aligned}
& \frac{\tau_{I}}{K_{c}} \frac{d \hat{f}_{\text {med }}}{d t}=K_{p} \sqrt{m_{h}^{2}}-\sqrt{\overline{m_{l}^{2}}}+K_{D} \frac{d\left[\sqrt{m_{h}^{2}}-\sqrt{m_{l}^{2}}\right]}{d t} \\
& \tau_{R} \frac{d \overline{m_{h}^{2}}}{d t}+\overline{m_{h}^{2}}=A^{2}\left[f_{h}-\hat{f}_{\text {med }}\right] \\
& \tau_{R} \frac{d \overline{m_{l}^{2}}}{d t}+\overline{m_{l}^{2}}=A^{2} \hat{f}_{\text {med }}
\end{aligned}
$$

where the variables and constants are listed in Table $I$. 


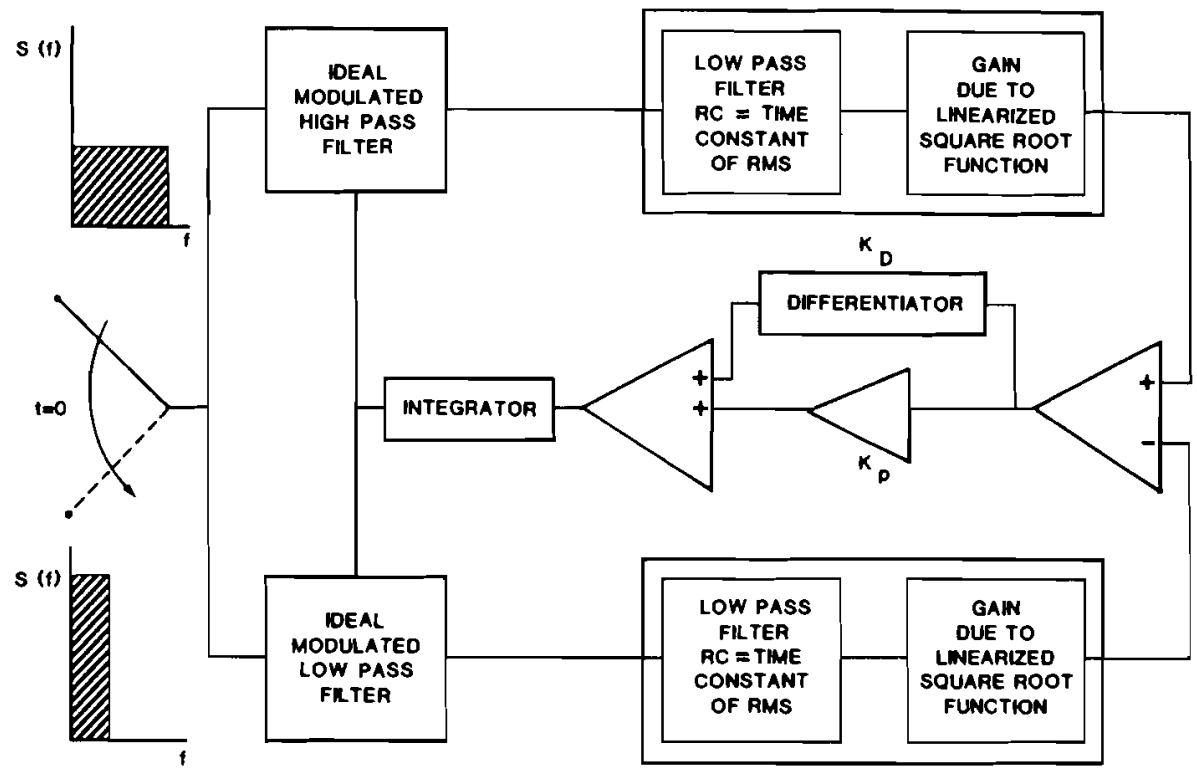

Fig. 9. Idealized and linearized dynamic model of the muscle fatigue monitor for minimizing the speed of response to an abrupt change in the spectral characteristics of the input signal.

TABLE I

Variables and Constants Used in the Optimization of the Speed of Response of the Muscle Fatigue Monitor

\begin{tabular}{|c|c|c|}
\hline $\begin{array}{l}\text { VARIABLE } \\
\text { OR } \\
\text { CONSTANT }\end{array}$ & DESCRIPTION & $\begin{array}{l}\text { UNITS } \\
\text { (Numbers in pareneneses } \\
\text { indicate as sumed values) }\end{array}$ \\
\hline A & Arbitrary Signal Level & $\begin{array}{l}\text { volts/Hertz } \\
(.01)\end{array}$ \\
\hline$f_{h}$ & Bandwidth of Signal & $\begin{array}{l}\text { Hertz } \\
(t=0-; 200) \\
t=0+; 100)\end{array}$ \\
\hline$\hat{\mathrm{f}}_{\text {med }}$ & Estimate of the Median Frequency & Kertz \\
\hline$k_{c}$ & $\begin{array}{l}\text { Proportionality Constant Between } \\
\text { the Median Frequency and Output } \\
\text { of the integrator }\end{array}$ & $\begin{array}{l}\text { Hertz/volt } \\
(15,0)\end{array}$ \\
\hline$k_{p}$ & Gain of Difference Amplifier & unitless \\
\hline$K_{D}$ & Gain of Differentiator Circuit & seconds \\
\hline$\overline{m^{2}}{ }_{h}$ & $\begin{array}{l}\text { Power Contained in signal } \\
\text { Between } F_{\text {med }} \text { and } f_{n}\end{array}$ & volts ${ }^{2}$ \\
\hline$\overline{m^{2}}$ & $\begin{array}{l}\text { Power Contained in Signal } \\
\text { Between } 0 \text { and } \mathrm{F}_{\text {med }}\end{array}$ & volts 2 \\
\hline${ }^{\tau} I$ & Time Constant of Integrator & $\begin{array}{l}\text { seconds } \\
(1.0)\end{array}$ \\
\hline$\tau_{R}$ & Time Constant of RMS circuits & seconds \\
\hline
\end{tabular}

The square root functions in (A2) are replaced by the linear portion of (A1). Taking the Laplace transforms, the following expression for the estimate of the median frequency is obtained:

$\hat{F}_{\text {med }}(s)=\frac{\left(A^{2} K_{c} / 2 \tau_{I} \tau_{R}\right)\left(K_{p}-K_{D} s\right) F_{h}(s)+\left(s+1 / \tau_{R}\right) \hat{f}_{\text {med }}^{o}}{s^{2}+\left[\left(1 / \tau_{R}\right)-\left(A^{2} K_{c} K_{D} / \tau_{I} \tau_{R}\right)\right] s+A^{2} K_{c} K_{p} / \tau_{I} \tau_{R}}$

The Laplace transforms of variables are indicated by capital letters. The factor $\hat{f}_{\text {med }}^{o}$ is the initial estimate of the value of the median frequency before the change in spectrum. It corresponds to the initial condition of the integrator. The input signal to drive the system is the step change in bandwidth of the spectrum modeled as a step change in $f_{h}(s)$.

The inverse Laplace transform of this expression was used to obtain the time-dependent estimate of the median frequency which would be determined by the MFM. The error between this estimate and the true median frequency $\left(f_{\text {med }}=f_{h} / 2\right.$ for the model signal) was used in an optimization program to select the gains $\left(K_{P}, K_{D}\right)$ of the control circuitry.

\section{ACKNOWLEDGMENT}

The authors wish to thank Dr. R. S. LeFever for his valued assistance in several details of the circuit design.

\section{REFERENCES}

[1] H. Piper, Electrophysiologie Muschliche Muskeln. Berlin, Germany: Springer-Verlag, 1912, p. 126.

[2] S. Cobb and A. Forbes, "Electromyographic studies of muscle fatigue in man," Amer. J. Physiol., vol. 65, pp. 234-251, 1923.

[3] K. Kogi and T. Hakamada, "Frequency analysis of the surface electromyogram in muscle fatigue," J. Sci Labour (Tokyo), vol. 38, pp. 519-528, 1962.

[4] R. Kadefors, E. Kaiser, and I. Petersén, "Dynamic spectrum analysis of myo-potentials with special reference to muscle fatigue," Electromyography, vol. 8, pp. 39-74, 1968.

[5] E. Kwatny, D. H. Thomas, and H. G. Kwatny, "An application of signal processing techniques to the study of myoelectric signals," IEEE Trans. Biomed. Eng., vol. BME-17, pp. 303-313, Oct. 1970.

[6] L. Lindström, R. Magnusson, and I. Petersén, "Muscular fatigue and action potential conduction velocity changes studies with frequency analysis of EMG signals," Electromyography, vol. 10, pp. 341-356, 1970.

[7] L. Lindström, R. Kadefors, and I. Petersén, "An electromyographic index for localized muscle fatigue," J. Appl. Physiol.: Respirat. Environ. Exercise Physiol., vol. 43, pp. 750-754, 1977.

[8] J.H.T. Viitasalo and P. V. Komi, "Signal characteristics of EMG during fatigue," Eur. J. Appl. Physiol., vol. 37, pp. 111-121, 1977.

[9] F. B. Stulen, "A technique for monitoring localized muscular fatigue using frequency domain analysis of the myoelectric signal," Ph.D. dissertation, Massachusetts Inst. Technol., Cambridge, MA, 1980.

[10] E. Stälberg, "Propagation velocity in human muscle fibers in situ," Acta Physiol. Scan., vol. 70, suppl. 287, 1966. 
[11] J. T. Mortimer, R. Magnusson, and I. Petersén, "Conduction velocity in ischemic muscle: Effect on EMG frequency spectrum," Amer. J. Physiol., vol. 219, pp. 1324-1329, 1970.

[12] B. Ahlborg, J. Bergstrom, L. G. Ekelund, G. Guarnieri, R.C. Harris, E. Hultman, and L. O. Nordesjo, "Muscle metabolism during isometric exercise performed at constant force," J. Appl. Physiol., vol. 33, pp. 224-228, 1972.

[13] N. P. Couch, J. R. Dmochowski, J. M. Van de Water, D. E. Harken, and $\mathrm{F}$. D. Moore, "Muscle surface $\mathrm{pH}$ as an index of peripheral perfusion in man," Ann. Surg., vol. 173, pp. 173-183, 1971.

[14] J. T. Mortimer, M. D. Kerstein, R. Magnusson, and I. Petersén, "Muscle blood flow in the human biceps as a function of developed muscle force," Arch. Surg., vol. 103, pp. 376-377, 1971

[15] R.H.T. Edwards, D. K. Hill, and M. McDonnell, "Myothermal and intramuscular pressure measurements during isometric contractions of the human quadriceps muscle," J. Physiol., vol. 224, pp. 58P-59P, 1972.

[16] J. Karlsson, "Muscle ATP, CP, and lactate in submaximal and maximal exercise," in Advances in Experimental Medicine and Biology, Vol. II: Muscle Metabolism During Exercise, B. Pernow and B. Saltin, Eds. Elmsford, NY: Pergamon, 1971.

[17] K. Sahlin, R. C. Harris, and E. Hultman, "Creatine kinase equilibrium and lactate content compared with muscle $\mathrm{pH}$ in tissue samples obtained after isometric exercise," Biochem. J., vol. 152, pp. 173-180, 1975.

[18] S. Rodbard and E. G. Pragay, "Contraction frequency, blood supply, and muscle pain," J. Appl. Physiol., vol. 24, pp. 142-145, 1968.

[19] R. Kadefors, I. Petersén, and P. Herberts, "Muscular reaction to welding work," Ergonomics, vol. 19, pp. 543-558, 1976.

[20] P. Herberts, R. Kadefors, and H. Broman, "Localized muscle fatigue in shoulder muscles: A preliminary study employing the spectral moment analyzer," in Proc. 4th Cong. Int. Soc. Electrophysiol. Kinesiol., Aug. 1979, pp. 72-73.

[21] M. Hagberg, "The elevated arm: Myoelectric amplitude and spectral changes in some shoulder muscles," in Proc. 4th Cong. Int. Soc. Electrophysiol. Kinesiol., Aug. 1979, pp. 70-71.

[22] H. O. Kendall, F. P. Kendall, and G. E. Wadworth, Muscles Testing and Function, Ed. 2. Baltimore, MD: Williams and Wilkins, 1971.

[23] R.H.T. Edwards and S. Hyde, "Methods of measuring muscle strength and fatigue," Physiotherapy, vol. 63, pp. 51-55, 1977.

[24] F. Bellemore and A. Grassino, "The fatigue and recovery of the human diaphragm," in Proc. 4th Cong. Int. Soc. Electrophysiol. Kinesiol., Aug. 1979, pp. 74-75.

[25] D. Gross, A. Grassino, W.R.D. Ross, and P. T. Macklem, "Electromyogram pattern of diaphragmatic fatigue," J. Appl. Physiol., vol. 46, pp. 1-7, 1979.

[26] D. Gross, H. Ladd, E. J. Riley, P. T. Macklem, and A. Grassino, "The effect of training on strength and endurance on the diaphragm in quadriplegia," Amer. J. Med., vol. 68, pp. 27-34, Jan. 1980.

[27] T. W. Schweitzer, J. W. Fitzgerald, J. A. Bowden, and P. LynneDavies, "Spectral analysis of human inspiratory diaphragmatic electromyograms," J. Appl. Physiol., vol. 46, pp. 152-165, 1979.

[28] S. Solomon, H. W. Ladd, B. C. Bradley, and P. T. Macklem, "A long-term electromyographical evaluation of inspiratory function in quadraplegic patients," in Proc. 4th Cong. Int. Soc. Electrophysiol. Kinesiol., Aug. 1979, pp. 92-93.

[29] F. B. Stulen and C. I. De Luca, "Frequency parameters of the myoelectric signal as a measure of muscle conduction velocity," IEEE Trans. Biomed. Eng., vol. BME-28, pp. 515-523, July 1981.

[30] -, "Monitoring myoelectric signals," U.S. Patent 4213467 , July 22,1980 .

[31] F. B. Stulen, "Monitoring myoelectric signals," U.S. Patent 4213466 , July 22,1980 .

[32] C. J. De Luca and W. Berenberg, "A polar technique for displaying EMG signals," in Proc. 28th Annu. Conf. Eng. Med. Biol., 1975 , p. 21
[33] A. L. Oslan, "Design and evaluation of a clinical muscle fatigue monitor," M.S. thesis, Boston Univ., Boston, MA, Apr. 1981.

[34] L. E. Franks and I. W. Sandberg, "An alternative approach to the realization of network transfer functions: The $N$-path filter," Bell Syst. Tech. J., pp. 1321-1350, 1960.

[35] G. Weiss, "Chopper network analysis," Polytechnic Inst. Brooklyn, Brooklyn, NY, Memo 5, R-708-58, PIB-6.36, 1956.

[36] H. C. Lin, W. R. Davis, and Y. Sun, "Active and passive multiplication of $R C$ time constants for subaudio frequency integrated filters," IEEE Region 6th Annu. Conf. Rec., 1966, vol. 1, pp. 1-9.

[37] Y. Sun and I. T. Frisch, "Resistance multiplication in integrated circuits by means of switching," IEEE Trans. Circuit Theory, vol. CT-15, pp. 184-192, Sept. 1968.

[38] J. A. Kaehler, "Periodic-switched filter networks-A means of amplifying and varying transfer functions," IEEE J. Solid-State Circuits, vol. SC-4, pp. 225-230, Aug. 1969.

[39] G. P. Edwards, "Variable-bandwidth filters using switching," Electron. Lett., vol. 5, p. $12,1969$.

[40] N. J. Hogan, "Myoelectric prosthesis control: Optimal estimation applied to EMG and the cybernetic considerations for its use in a man-machine interface," Ph.D. dissertation, Massachusetts Inst. Technol., Cambridge, MA, Aug. 1976.

[41] C. J. De Luca, R. S. LeFever, and F. B. Stulen, "Pasteless electrode for clinical use," Med. Biol. Eng. Comput., vol.17, pp. 387$390,1979$.

[42] F. B. Stulen and C. J. De Luca, "The relation between the myoelectric signal and physiological properties of constant force isometric contractions," Electroencephogr. Clin. Neurophysiol., vol. 45 , pp. $681-698,1978$.

[43] H. Broman and R. Kadefors, "A spectral moment analyzer for quantification of electromyograms," in Proc. 4th Cong. Int. Soc. Electrophysiol. Kinesiol., Aug. 1979, pp. 90-91.

[44] J. S. Petrofsky, "Filter bank analyser for automatic analysis of the EMG," Med. Biol. Eng. Comput., vol. 18, pp. 585-590, 1980.

[45] P. A. Lynn, "Direct on-line estimation of muscle fiber conduction velocity by surface electromyography," IEEE Trans. Biomed. Eng., vol. BME 26, pp. 564-571, Oct. 1979.

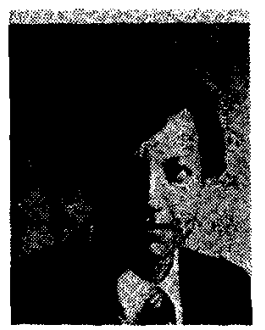

Foster B. Stulen (S'78-M'79) was born in Morristown, $\mathrm{NJ}$, on November 3,1951 . He received the B.S. degree in mechanical engineering in 1973 from Rensselaer Polytechnic Institute, Troy, NY, and the S.M. and Ph.D. degrees in mechanical engineering from Massachusetts Institute of Technology, Cambridge, in 1975 and 1980 , respectively.

He worked as a consultant in bioelectric signal processing to the Children's Hospital Medical Center, Boston, MA, during 1979. He is currently a Research Scientist in the Applied Dynamics and Acoustics Section of Battelle Columbus Laboratories, Columbus, $\mathrm{OH}$. His research activities there involve the application of both high- and low-intensity ultrasonics in the areas of nondestructive testing, leak detection in nuclear piping and boilers, and material bonding. He is also involved in the field of acoustic emission for flaw detection in complex and large structures.

Dr. Stulen is a member of the American Society of Mechanical Engineers and Sigma Xi.

Carlo J. De Luca (S'64-M'72-SM'77), for a photograph and biography, see p. 157 of the March 1982 issue of this TRANSACtIons. 